Ab initio quantum dynamics with very weak van der Waals interactions: Structure and stability of small $\mathrm{Li}_{2}\left({ }^{1} \Sigma_{g}^{+}\right)-(\mathrm{He})_{n}$ clusters

E. Bodo, F. Sebastianelli, and F. A. GianturcoE. YurtseverM. Yurtsever

Citation: The Journal of Chemical Physics 120, 9160 (2004); doi: 10.1063/1.1701834

View online: http://dx.doi.org/10.1063/1.1701834

View Table of Contents: http://aip.scitation.org/toc/jcp/120/19

Published by the American Institute of Physics

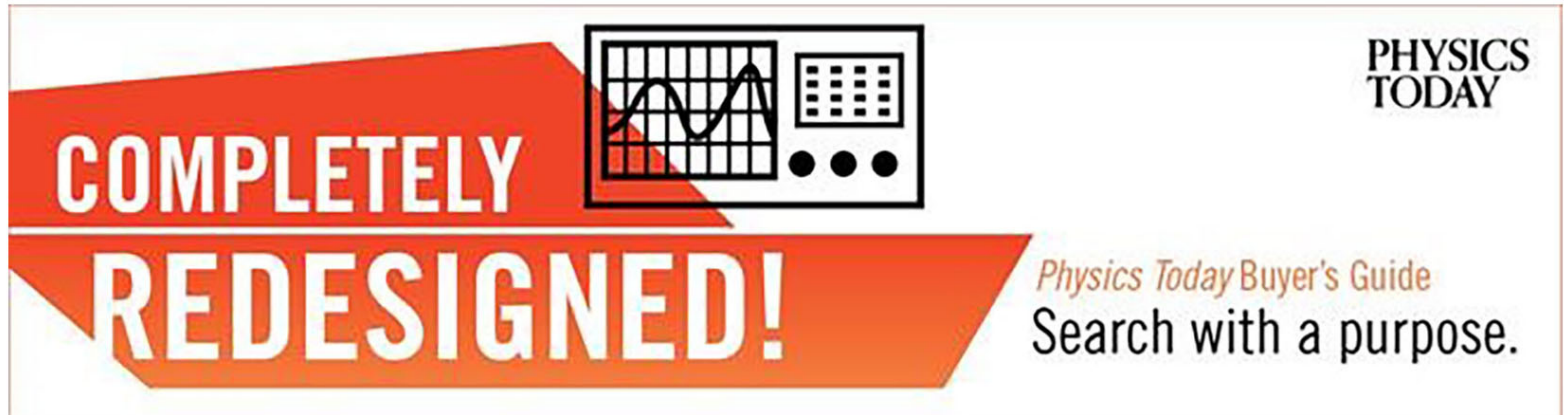




\title{
Ab initio quantum dynamics with very weak van der Waals interactions: Structure and stability of small $\mathrm{Li}_{2}\left({ }^{1} \Sigma_{g}^{+}\right)-(\mathrm{He})_{n}$ clusters
}

\author{
E. Bodo, F. Sebastianelli, and F. A. Gianturco ${ }^{\text {a) }}$ \\ Department of Chemistry and INFM, University of Rome "La Sapienza," Italy \\ E. Yurtsever \\ Department of Chemistry, Koç University, Istanbul, Turkey \\ M. Yurtsever \\ Department of Chemistry, Technical University of Istanbul, Turkey
}

(Received 4 December 2003; accepted 20 February 2004)

\begin{abstract}
The potential energy surface (PES) for the interaction between $\operatorname{Li}_{2}\left({ }^{1} \Sigma_{g}^{+}\right)$and ${ }^{4} \mathrm{He}$ has been computed using an accurate, post-Hartree-Fock quantum calculation for its ground electronic state. The orientational anisotropy of the forces and the interplay between repulsive and attractive effects within the PES are analyzed to extract information on the possible existence of bound states in the triatomic system. The structures of a few of the $\mathrm{Li}_{2}(\mathrm{He})_{n}$ small clusters are examined by comparing a classical approach with a full quantum one to generate bound configurations and to extract information on the possible spatial arrangements of the smaller clusters via à vis the location of the $\mathrm{Li}_{2}$ dopant. Some significant consequences on the $\mathrm{Li}_{2}$ behavior in larger clusters and droplets are drawn from the above findings. (C) 2004 American Institute of Physics. [DOI: 10.1063/1.1701834]
\end{abstract}

\section{INTRODUCTION}

The accurate evaluation of the interaction potential between a closed-shell molecule and a single ${ }^{4} \mathrm{He}$ atom is a key step in modeling the general behavior of small-size doped helium clusters because it can be used to set up the total potential acting within them, an essential first step for determining their structure and dynamics. Nanosized helium clusters or droplets in which a molecular or an atomic impurity has been added by means of controlled solvation techniques provide a unique environment for high-precision spectroscopic studies of the molecular solute that would be otherwise impossible to obtain in bulk helium. ${ }^{1-3}$ Among the many dopants that have been experimentally and theoretically analyzed, the alkali metals have many interesting properties. In most cases, a closed-shell molecule or an atom is rather strongly bound to the droplet: it remains inside the droplet and becomes surrounded by a slightly compressed solvation shell of helium atoms. Since the interaction between the molecule and helium atoms is in general anisotropic, the nonuniform angular distribution of the solvation shell gives rise to a rich variety of "geometric" configurations (in a picture where atoms are classical points) in the vicinity of the dopant. In the case of an alkali atom the situation is different: from experimental ${ }^{4}$ and theoretical ${ }^{5}$ evidence it turns out that they normally reside on the surface of the droplet, usually forming a slight "dimple" on that surface. This is probably due to the repulsive interaction between the outer, unpaired electron of the alkali dopants and the closed shells of the surrounding helium atoms. When more alkali atoms are added to the droplet they form (with-

\footnotetext{
a) Author to whom correspondence should be addressed. Fax: +39-0649913305. Electronic mail: fa.gianturco@ caspur.it
}

out any kinetic barrier) a singlet or a triplet molecule that still resides on the surface. The singlet molecules have been detected by electronic spectroscopy, but the internal relaxation following their formation leads to a rapid detachment of them from the droplet and therefore to weak emission spectra: $^{6}$ they bind too weakly to the cluster and therefore undergo evaporation rather easily. In contrast, the bound triplet molecules manage to cool to the droplet temperature by remaining on the surface, thereby making their detected spectra much stronger. ${ }^{6,7}$ It would therefore be interesting to analyze from the theoretical standpoint the general and specific features of the alkali-molecule-He interactions, to examine the energy transfer between them, and to predict the structure of the solvation shells that one could obtain from accurate $a b$ initio calculations.

There have been in the past various experimental studies on the $\mathrm{Li}_{2}-\mathrm{He}$ system that have examined the influence of the vibrational energy content of $\mathrm{Li}_{2}$ molecules in collision with rare-gas atoms ${ }^{8}$ when producing initial-state-selected integral cross sections. Various theoretical studies employed either ab initio or semiempirical potentials (see, for example, Refs. 9 and 10 and references therein) or used the results of the experiments to model the anisotropic interaction potential for $\mathrm{Li}_{2}-\mathrm{He}$ (Ref. 11). The present approach has chosen instead to evaluate the rigid rotor (RR) interaction using a highly correlated ab initio approach.

We will describe in the next section some details of the computation of an accurate RR potential energy surface (PES) for the title system, while in Sec. III we will show the behavior of the very-low-energy elastic cross sections and the features of the scattering length yielded by the RR potential for the examined collision events. Finally, we will report in Sec. IV a series of ab initio results and of stochastic calculations that provide the most likely structures of the 


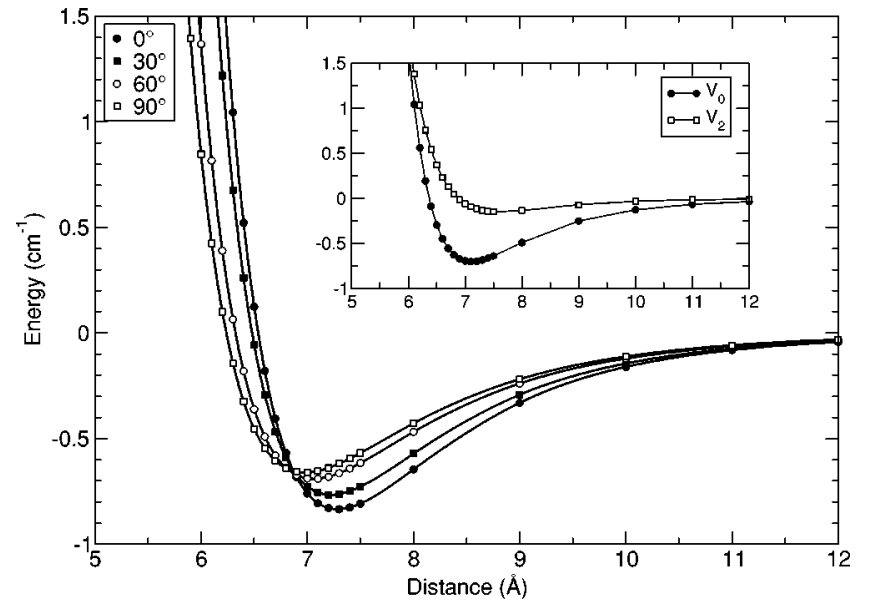

FIG. 1. Computed interaction as a function of $R$ and for four different angular orientations. The inset reports the first two expansion terms $V_{0}$ and $V_{2}$.

small, bound clusters of $\mathrm{Li}_{2}$ with a few ${ }^{4} \mathrm{He}$ atoms, for which we will endeavor to suggest a possible model for the organization of their first solvation shell. Internal Jacobi coordinates, $\AA$ for distances, and $\mathrm{cm}^{-1}$ for energies are used throughout this paper.

\section{RIGID ROTOR POTENTIAL}

The PES has been computed in Jacobi coordinates fixing the internuclear distance of the $\mathrm{Li}_{2}$ molecule at its equilibrium value of $2.7 \AA$. The extension of the calculations to other $\mathrm{Li}_{2}$ geometries is currently in progress and will be analyzed elsewhere. We believe, however, that the present computed interaction should be accurate enough to reliably perform the studies described below, since the He atom turns out to be a very weak perturber of the molecule.

The $a b$ initio calculations have been done via GAUSSIAN 98 (Ref. 12), using the QCISD(T) method with a cc-pvQZ basis set and with a frozen lithium core. Each of the calculated points has been counterpoise corrected to avoid the basis set supposition errors (BSSEs) that turn out to be very large in this system. The calculations have been repeated for selected geometries with the $\operatorname{CCSD}(\mathrm{T})$ method, but using the same basis set: the computed energies are the same within $10^{-6} \%$. For another set of selected geometries computations have been repeated without freezing the lithium core, but no more than $2 \%-3 \%$ differences were obtained with respect to the previous results. More details on the $a b$ initio approach as well as the specific form of an analytical fitting of the PES employed here can be found in Ref. 13.

The produced molecular interaction turns out to be essentially isotropic, as further confirmed when one looks at the usual multipolar expansion coefficients

$$
V_{\lambda}=\frac{1}{2 \lambda+1} \int_{-1}^{1} V(R, \theta) P_{\lambda}(\cos \theta) d \cos \theta,
$$

which we found to be numerically converged when using the largest multipolar coefficient with $\lambda=6$. As a pictorial example, the first two terms of the $V_{\lambda}$ expansion are reported in the inset of Fig. 1. The behavior minimum energy angular

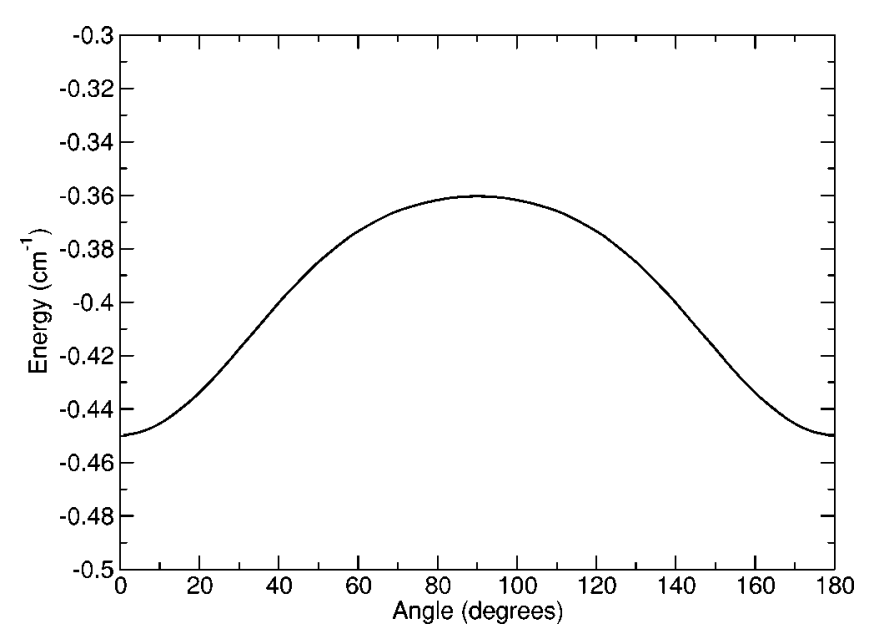

FIG. 2. Minimum-energy angular path.

path for the title system is reported in Fig. 2 as a function of the Jacobi angle: it clearly shows how small the orientational variation of the PES calculated in the present work is.

In Ref. 13 we have made a detailed comparison with earlier potential energy surfaces ${ }^{8,9,11}$ in order to get a better understanding of the overall reliability of our results. The potentials obtained by Rubahn ${ }^{9}$ were found to be, in general, weaker with respect to ours. The model potential put together by Fuchs and Toennies ${ }^{8}$ showed instead a well region which was much deeper than ours. Although the present PES and previous empirical estimates differ between each other, it is nevertheless true for all existing PES's that they exhibit rather weak angular anisotropies, a feature that was indeed important for the analysis of the rotational deexcitation collisions at ultralow energies already reported by us for the present system ${ }^{13}$ where we found that the computed energytransfer probabilities suggested a marked inefficiency of the collisional cooling mechanism. To get a more precise idea of the global behavior of this new PES we also report a threedimensional (3D) view of it in Fig. 3. In that picture one can easily notice the chiefly isotropic shape of the interaction and the well-defined molecular core containing the $\mathrm{Li}_{2}$ partner, identified by the repulsive region which gives a negligible indication of any orientation-dependent molecular feature.

\section{ULTRALOW-ENERGY ELASTIC CROSS SECTIONS AND BOUND STATES}

Although this work is chiefly focused on the structure and energetics of the $\mathrm{Li}_{2}-(\mathrm{He})_{n}$ complexes, to better understand the unusual features of the forces at play in such systems we report, albeit in a condensed form, some of the dynamical attributes which have been presented in Ref. 13. The analysis of the elastic cross sections for the collision of $\mathrm{Li}_{2}$ with one helium atom may indeed be able to tell us whether or not a bound state exists for the triatomic complex $\mathrm{Li}_{2}-\mathrm{He}$. For such weakly interacting system, in fact, the limiting values of the $S$ matrix for zero collision energy is able to yield, within a given accuracy, the possible location of its bound states. As a test case, we have used the diatomic ${ }^{7} \mathrm{Li}-{ }^{4} \mathrm{He}$ molecule for which we have obtained the binding energy of $-0.0012 \mathrm{~cm}^{-1}$ using the potential reported in Ref. 


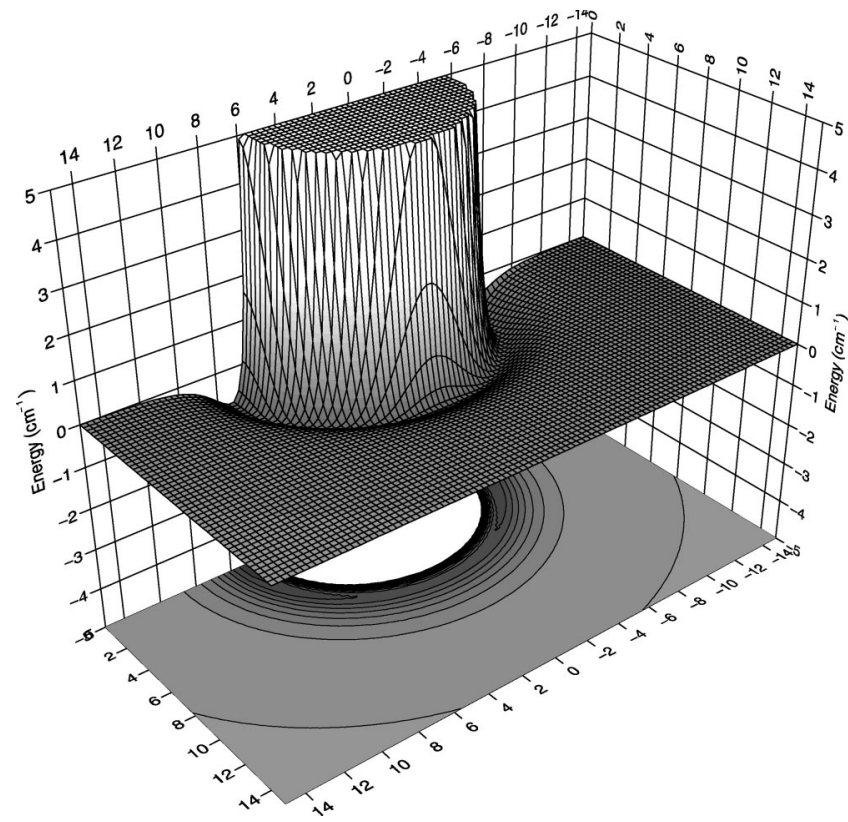

FIG. 3. Three-dimensional view of the interaction potential. Energies in $\mathrm{cm}^{-1}$ and distances in $\AA$.

14 that has to be compared with the value $-0.0015 \mathrm{~cm}^{-1}$ reported in Ref. 15 or the value $-0.00195 \mathrm{~cm}^{-1}$ of Ref. 16 .

In the case of an elastic collision between $\operatorname{Li}_{2}(j=0)$ and ${ }^{4} \mathrm{He}$, the scattering length can be calculated rather directly from the elastic cross section (for a detailed discussion see Ref. 13). For a potential with such a small attractive well and that vanishes asymptotically at least as $R^{-3}$, one bound state exists for the triatomic system only if the scattering length is positive. Elastic cross sections have been calculated by using the time-independent approach to multichannel scattering and by solving the coupled-channels equations. In the limit of zero collision energy the cross section for the collision between the $\mathrm{Li}_{2}$ molecule and an ${ }^{4} \mathrm{He}$ atom is $\sim 716 \AA$. Such a large value attained by the cross section at ultra low collision energies is due to a pole in the complex energy plane of the corresponding $S$ matrix that lies near the continuum threshold. The energy of this pole can be calculated ${ }^{17}$ and turns out to be $\sim-0.34 \mathrm{~cm}^{-1}$. The scattering length obtained from the elastic $S$ matrix that was yielded by the above calculations was found to be negative, and therefore the above energy corresponds to a "virtual state." We can thus conclude that, at least when using our ab initio PES, the $\mathrm{Li}_{2}-\mathrm{He}$ system does not have any physical bound state.

\section{ENERGIES AND STRUCTURES OF THE SMALLER $\mathrm{Li}_{2}(\mathrm{He})_{n}$ AGGREGATES}

\section{A. Ab initio structure optimization and many-body contributions}

Since the $\mathrm{Li}_{2}-\mathrm{He}$ moiety appears to be not bound (within the interaction potential calculated here), we have further extended our study to clusters containing more than one ${ }^{4} \mathrm{He}$ atom. The well-known problem in such weakly interacting systems is how to deal correctly with the large zeropoint energy (ZPE) exhibited by the physical many-body bound states. The simple construction of the possible "clas- sical" structures provided via the minimization of the total $a b$ initio energy is seldom sufficient to describe the highly quantum nature of the atoms that form such systems. We will therefore present below an analysis of the minimum-energy structures which have been obtained from conventional quantum chemical geometry optimizations and which we shall call as being the "classical" structures of these aggregates. We will also discuss more correct quantum calculations of the ZPE values which have been obtained from a quantum diffusion Monte Carlo (DMC) approach to get the bound wave functions of the clusters and their lowest states associated to these nodeless quantum structures.

We have employed two different approaches to the calculation of the quantum chemical energy minima on the multidimensional surfaces. The first one is based on the full $a b$ initio calculation of the interaction energy: the second calculates, instead, the total potential as a sum of two-body (2B) interactions:

$V\left[\mathrm{Li}_{2}-(\mathrm{He})_{n}\right]=\sum_{i}^{n} V\left[\mathrm{Li}_{2}-\mathrm{He}_{i}\right]+\sum_{i<j}^{n} V\left[\mathrm{He}_{i}-\mathrm{He}_{j}\right]$,

where $i$ and $j$ run over the number of helium atoms in each system. The former approach is complicated by the fact that we have to take into account the BSSE correction to the interaction energy as we did for the PES described above. The structure optimization was carried out using the MP4 method. We are further aware of the fact that, at our level of calculation, the correct interaction energy of two helium atoms is underestimated by about a factor of 2 . We therefore thought it more correct to confirm the geometries of the resulting structures using the data from a sum of two-body potential functions where the $2 \mathrm{~B}$ forces describe fairly realistically the interactions between the helium atoms. It is worth pointing out, in fact, that the $2 \mathrm{~B}$ approximation for the calculation of the total potential is based on the assumption that three-body forces are extremely small, as is often the case for weakly interacting closed-shell systems like those we are considering in the present work.

The full ab initio minimization procedure was performed in various steps: first, a geometry optimization using MP4 and a cc-pVTZ basis sets was employed to localize the "likely" structure for the energy minimum by simultaneously optimizing all the coordinates. Then, the interatomic distances were varied while preserving the symmetry of the optimized geometry and the various total energies were then calculated using the same approach and a cc-pVQZ basis set. The full counterpoise correction was then added to ensure that the geometry remained at a minimum of the energy after the correction (or at least very near to a minimum). We have followed the above procedure to optimize the structures for $\mathrm{Li}-(\mathrm{He})_{n}$ with $n=2,3,4,5$, and 6 . Various initial minima have been analyzed by us, although in Fig. 4 we report only the three structures which exhibited the lowest energy within each cluster. The resulting complexes are highly "one-sided" in terms of helium atoms and show clearly the incipient formation of a pure $\mathrm{He}$ cluster with the $\mathrm{Li}_{2}$ molecule sitting on its "surface." This result seems to confirm the experimental suggestions ${ }^{4}$ and the earlier theoretical predictions of Ref. 5 

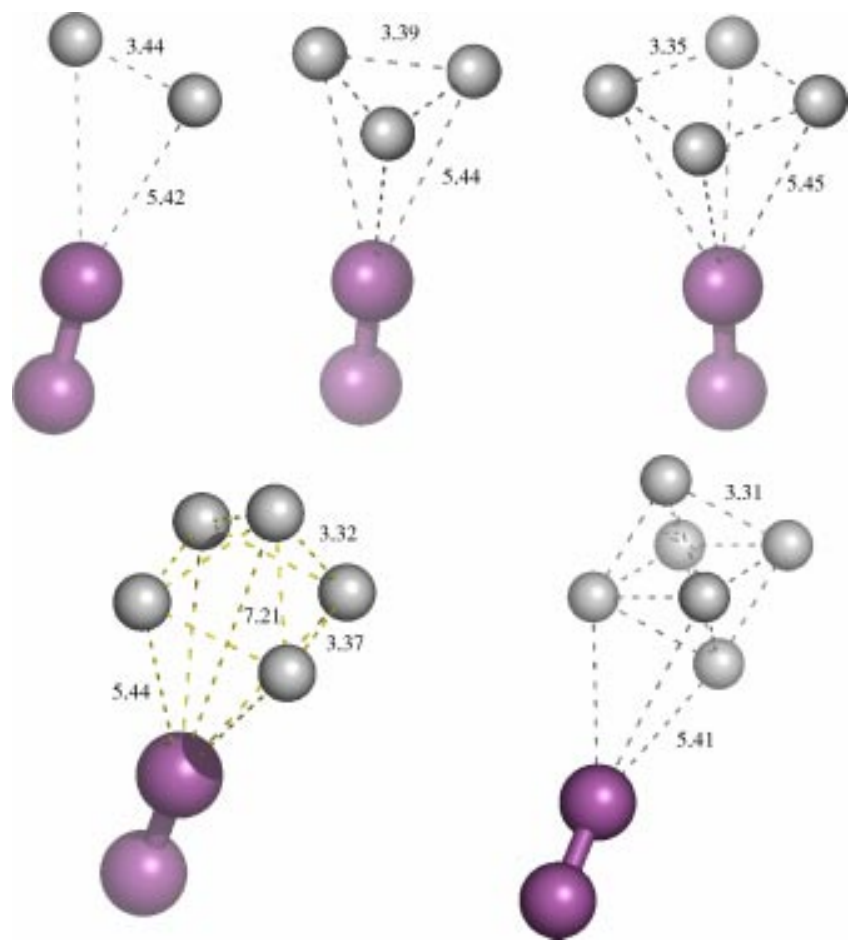

FIG. 4. Lowest energy full ab initio structures for $\mathrm{Li}-(\mathrm{He})_{n}$, for $n$ from 2 to 6. Distances are in $\AA$.

which localized the molecule outside the droplet. In other words, since the $\mathrm{Li}_{2}-\mathrm{He}$ interaction is weaker than that between the atomic partners, the molecule is not solvated by the ${ }^{4} \mathrm{He}$ atoms, which tend instead to preferentially form pure helium clusters that are only slightly modified in structures and in binding energies by the presence of an outside $\mathrm{Li}_{2}$ impurity positioned fairly far from them.

In addition, as we have mentioned earlier, the reliability of the above structures has been checked through a minimization calculation which used a total potential obtained as the sum of two-body contributions where the $\mathrm{Li}_{2}$ dopants is treated as a rigid rotor. ${ }^{18}$ The results from the $2 \mathrm{~B}$ potentials produced energy values and geometries which were very close to the quantum chemical results.

\section{B. Quantum Monte Carlo dynamics for structure evaluation}

The implementation of the diffusion Monte Carlo approach and its application to molecular systems such as molecular impurities in ${ }^{4} \mathrm{He}$ droplets has been already discussed, ${ }^{19-28}$ and we therefore only summarize the main features of the method employed here.

The Schrödinger equation can be written in the imaginary time $t=i \tau / \hbar$ :

$$
i \hbar \frac{\partial \psi(\mathbf{r}, t)}{\partial t}=(\hat{H}-E) \psi(\mathbf{r}, t)
$$

where $\mathbf{r}$ denotes collectively all the particle coordinates and $E$ shifts the absolute energy scale of the problem at hand. As we have said above, the Hamiltonian is made up from pairwise potentials

$$
\hat{H}=\sum_{j} \frac{\hbar^{2}}{2 m_{j}} \nabla_{j}^{2}+V(\mathbf{r})
$$

and we can use it in expression (2) to obtain the diffusion equation for the wave function:

$$
-\frac{\partial \psi(\mathbf{r}, t)}{\partial t}=-\sum_{j} D_{j} \nabla_{j}^{2} \psi(\mathbf{r}, t)+[V(\mathbf{r})-E] \psi(\mathbf{r}, t),
$$

where $D_{j}=\hbar^{2} / 2 m_{j}$ are the diffusion coefficients. The second term on the right-hand side (rhs) of Eq. (3) $[V(\mathbf{r})-E]$ is the "rate constant" or branching term. Ignoring the branching term transforms Eq. (3) into a simpler pure diffusion equation. It is well known that better efficiency in the solution of Eq. (3) is attained if one uses importance sampling that consists in constructing an analytical trial function $\psi_{T}(\mathbf{r}, t)$ which approximates the true ground-state wave function and then solving the diffusion equation (3) for the mixed distribution $f(\mathbf{r}, t)=\psi_{T}(\mathbf{r}, t) \psi(\mathbf{r}, t)$, which satisfies the equation

$$
\begin{aligned}
-\frac{\partial f(\mathbf{r}, t)}{\partial t}= & -\sum_{j}\left[D_{j} \nabla_{j}^{2} f(\mathbf{r}, t)+D_{j} \vec{\nabla} \cdot(\vec{F} \cdot f(\mathbf{r}, t))\right] \\
& +\left[E_{L}(\mathbf{r})-E\right] f(\mathbf{r}, t),
\end{aligned}
$$

where $\vec{F}=\psi_{T} \nabla \ln \psi_{T}$ is a drift force proportional to the gradient of the trial wave function pointing in the direction where $\psi_{T}$ increases and $E_{L}$ is the local energy defined as

$$
E_{L}(\mathbf{r})=\psi_{T}^{-1}(\mathbf{r}) \hat{H} \psi_{T}(\mathbf{r})=\psi_{T}^{-1}(\mathbf{r}) \hat{T} \psi_{T}(\mathbf{r})+V(\mathbf{r})
$$

A random walk technique is used to solve Eq. (4) where a large number of random walkers is propagated with time steps $\Delta \tau$ starting from an arbitrarily chosen initial distribution. The random displacements of the walkers when moving from $\tau$ to $\tau+\Delta \tau$ is subjected to the drift force and to a Metropolis-type acceptance algorithm..$^{20,22,23,27}$ The groundstate energy $E_{0}$ is obtained by averaging $E_{L}(\mathbf{r})$ over the final mixed distributions $f\left(\mathbf{r}, \tau_{f}\right)=f(\mathbf{r})$ as long as the trial function $\psi_{T}$ is not vanishing in spatial regions of significant contribution to the ground-state wave function $\psi_{0}$ :

$\left\langle E_{L}\right\rangle=\frac{\int E_{L}(\mathbf{r}) f\left(\mathbf{r}, \tau_{f}\right) d \mathbf{r}}{\int f\left(\mathbf{r}, \tau_{f}\right) d \mathbf{r}}=\frac{\int \psi_{0}(\mathbf{r}) \hat{H} \psi_{T}(\mathbf{r}) d \mathbf{r}}{\int \psi_{0}(\mathbf{r}) \psi_{T}(\mathbf{r}) d \mathbf{r}}=E_{0}$.

Expectation values of position operators $\hat{A}(\mathbf{r})$ are also given by averaging over $f(\mathbf{r})$. The Monte Carlo walk converts the integrals over $f(\mathbf{r})$ into simple averages over the ensemble of walkers $\left\{\mathbf{r}_{j}\right\}$ and their associated weights $\left\{w_{j}\right\}$ :

$$
\langle\hat{A}\rangle=\frac{\sum_{j} w_{j} \hat{A}\left(\mathbf{r}_{j}\right)}{\sum_{j} w_{j}} \text {. }
$$

Direct averaging over the weighted configurations can lead, however, to mixed average $\left\langle\psi|\hat{A}| \psi_{T}\right\rangle$, which can contain some bias for operators $\hat{A} \neq \hat{H}$.

The potential acting within each cluster was expressed as a sum of pairwise potentials as in Eq. (1) where we have used the He-He potential of Aziz et al. ${ }^{29}$ The trial function used here for the $\mathrm{He}-\mathrm{He}$ pairs is a product of the trial wave function: 
TABLE I. $\mathrm{Li}_{2}-\mathrm{He}_{n}$ cluster energies. All calculations using the DMC method discussed in the text. The pure cluster data from Ref. 30 (all quantities in $\left.\mathrm{cm}^{-1}\right)$.

\begin{tabular}{rllc}
\hline \hline$n$ & $E\left(\mathrm{Li}_{2}-\mathrm{He}_{n}\right)$ & $E\left(\mathrm{He}_{n}\right)$ & $E\left(\mathrm{Li}_{2}-\mathrm{He}_{n}\right)-E\left(\mathrm{He}_{n}\right)$ \\
\hline 3 & $-0.110 \pm 0.004$ & -0.0910 & $-0.019 \pm 0.004$ \\
4 & $-0.457 \pm 0.005$ & -0.4012 & $-0.056 \pm 0.005$ \\
5 & $-1.026 \pm 0.009$ & -0.928 & $-0.098 \pm 0.009$ \\
6 & $-1.79 \pm 0.012$ & -1.647 & $-0.14 \pm 0.012$ \\
7 & $-2.72 \pm 0.020$ & -2.533 & $-0.19 \pm 0.020$ \\
8 & $-3.78 \pm 0.027$ & -3.568 & $-0.21 \pm 0.027$ \\
9 & $-4.99 \pm 0.026$ & -4.731 & $-0.25 \pm 0.026$ \\
10 & $-6.29 \pm 0.027$ & -4.989 & $-0.27 \pm 0.027$ \\
\hline \hline
\end{tabular}

$$
\Psi_{T}=\prod_{i, j \in \mathrm{He}} \exp \left(-\frac{p_{5}}{R_{i j}^{5}}-\frac{p_{2}}{R_{i j}^{3}}-p_{0} \ln R_{i j}-p_{1} R_{i j}\right),
$$

where the values of the coefficients have been taken as in Ref. 30. The trial function for the $\mathrm{Li}_{2}-\mathrm{He}$ pair has been chosen as given by the same formula (the trial function is therefore isotropic) although we modified the various coefficients of above in the following fashion: $p 5=200000.0$, $p 2=0.600, p 0=0.500, p 1=0.005$. We have propagated the diffusion equations for 9000 atomic units of time using up to 375000 walkers to get converged distributions and energies.

For the smallest first two structures $\left(\mathrm{Li}_{2}-\mathrm{He}\right.$ and $\mathrm{Li}_{2}-\mathrm{He}_{2}$ ) our Monte Carlo calculations were not able to find a bound state, although the phase space sampled by our calculations was quite large $\left(\sim 80\right.$ a.u. $\left.{ }^{3}\right)$ since we were expecting marked atomic delocalizations in the resulting wave functions due to the large zero-point energy of each aggregate. The above result was somehow expected since it is just a confirmation of what the scattering calculations that we have reported in Sec. III had already told us.

The first cluster that appears to have at least one bound state when the present interaction potential is used turned out to be $\mathrm{Li}_{2}-\mathrm{He}_{3}$, but we have extended the calculations to larger $\mathrm{Li}_{2}-\mathrm{He}_{n}$ with $n$ up to 10 . The energies of the clusters analyzed here, together with their numerical uncertainty values, are reported in Table I and Fig. 5. The full binding

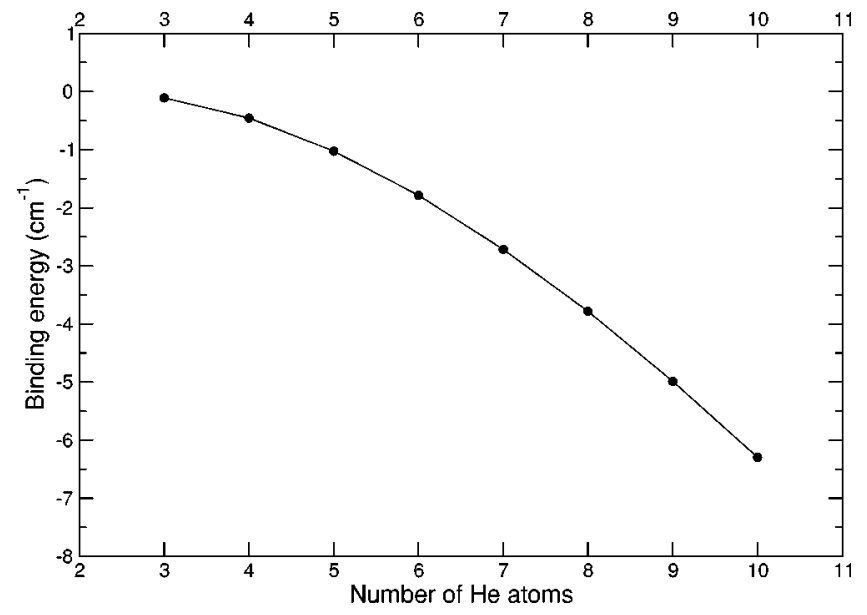

FIG. 5. DMC energies of the first $\mathrm{Li}_{2}-\mathrm{He}_{n}$ clusters. The computed error bars are smaller than the symbols reported in the figure.

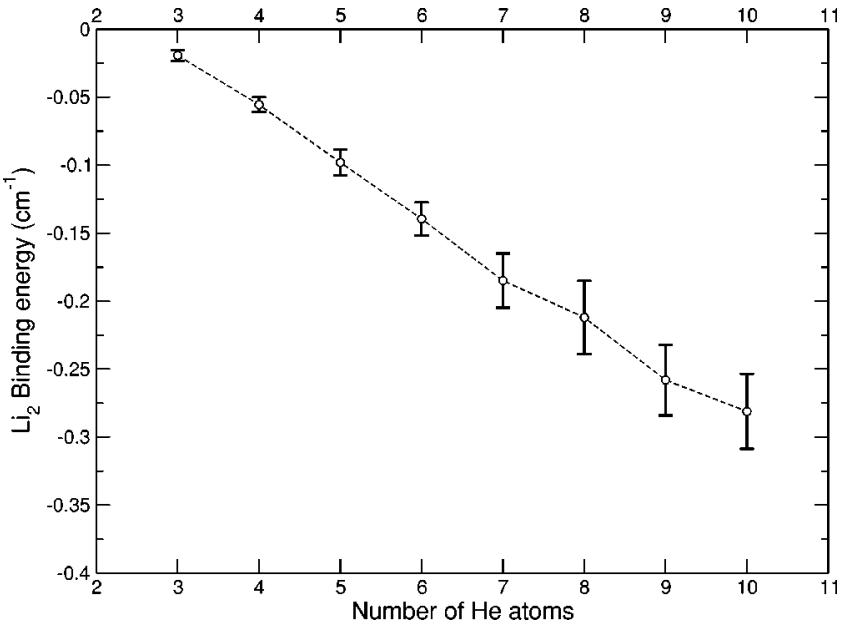

FIG. 6. Binding energies of the $\mathrm{Li}_{2}$ molecule calculated as $E\left(\mathrm{Li}_{2}-\mathrm{He}_{n}\right)$ $-E\left(\mathrm{He}_{n}\right)$ where the energies of the pure helium cluster have been taken from Ref. 30 .

energies that we have obtained are extremely small and are comparable with the energies of the corresponding pure $\mathrm{He}_{n}$ cluster reported in Table I (taken from the DMC results in Ref. 30). We were indeed already expecting the effect from the $\mathrm{Li}_{2}-\mathrm{He}$ interaction to be very small with respect to the $\mathrm{He}-\mathrm{He}$ energy contribution: by calculating the difference $E\left(\mathrm{Li}_{2}-\mathrm{He}_{n}\right)-E\left(\mathrm{He}_{n}\right)$ we can therefore estimate the binding energy of the $\mathrm{Li}_{2}$ molecule to the cluster (that is, the evaporation energy of the molecule from the droplet). These binding energies, reported for clarity also in Fig. 6, turn out to be extremely small: of the order of $0.1-0.2 \mathrm{~cm}^{-1}$ with an almost linear dependence on the number of atoms. From the energetics point of view, it therefore seems that the presence of the $\mathrm{Li}_{2}$ molecule adds only a very small perturbation to the energies that bind bosonic clusters of pure helium.

Various experimental and theoretical studies, as we have mentioned in the Introduction, have proposed structures for alkali-dimer-helium clusters in which the diatomic impurity resides on the droplet surface. It is, however, very difficult to establish a "structure" when the energy locations of the ground quantum states of the clusters give rise to values of ZPE which are such a large fraction of the total BornOppenheimer binding energies. The wave functions of the helium atoms and of the molecules are largely delocalized, and it therefore turns out to be nearly impossible to describe this kind of clusters in terms of conventional (classical) minimum-energy structures. However, a certain degree of understanding is still possible by analyzing the quantum probability distributions that we have obtained from the quantum DMC calculations described above.

The chief problem here is to establish whether the molecule is somehow contained inside the small droplet formed by the helium atoms or if it resides on the surface of an unperturbed, pure helium bosonic cluster. We believe that our full quantum mechanical calculations (at least those obtained when using the interaction potential presented in this work) lead to the latter picture. We begin the discussion by looking at the quantum mechanical radial probability distri- 

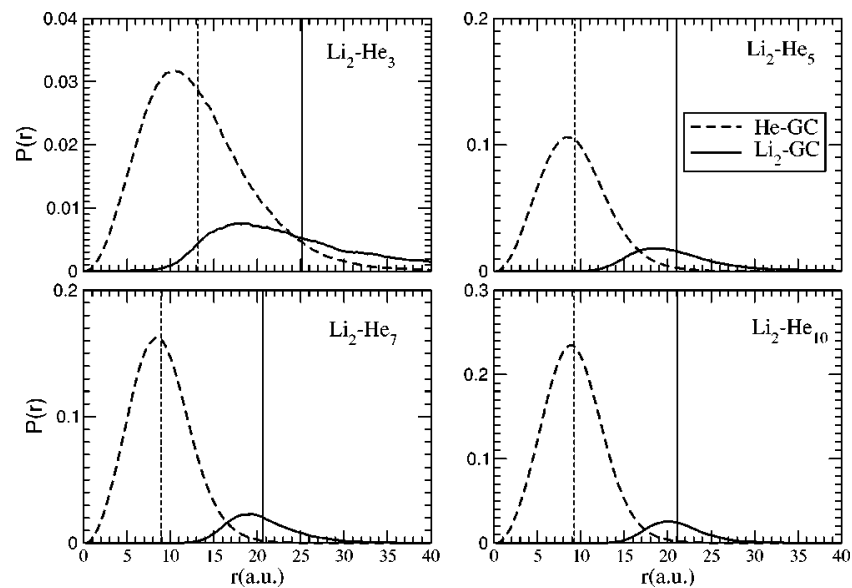

FIG. 7. Quantum mechanical radial distributions of the $\mathrm{He}$ atoms and $\mathrm{Li}_{2}$ molecule with respect to the geometrical center of the cluster. Distributions medians are also drawn superimposed as vertical lines. Distances are in a.u.

bution of the diatomic molecule and of the "solvent" $\mathrm{He}$ atoms calculated with respect to the geometrical center (GC) of the aggregate that is explicitly defined as

$$
\mathbf{r}_{\mathrm{GC}}=\frac{\sum_{i}^{n(\mathrm{He})} \mathbf{r}_{i}(\mathrm{He})+\mathbf{r}\left(\mathrm{Li}_{2}\right)}{n(\mathrm{He})+1}
$$

where $\mathbf{r}\left(\mathrm{He} / \mathrm{Li}_{2}\right)$ are the distances of the atoms and of the molecule from the center of the reference frame. The two distributions for selected sizes of the clusters $(n=3,5,7,10)$ are reported in Fig. 7. The He atoms are seen to be localized within a "first shell" that is spatially isotropic and centered around the GC, with an average distance from it of about $\sim 10$ a.u. As expected, when we increase the number of ${ }^{4} \mathrm{He}$ atoms, this distribution tends to get more localized because the total energy content of the cluster is increased. $\mathrm{The}^{\mathrm{Li}} \mathrm{i}_{2}$ molecule, with the exception of the first $\mathrm{Li}_{2}-\mathrm{He}$ structure, is instead localized over an outer shell placed around the geometric center and whose average distance from the helium shell turns out to be $\sim 10$ a.u.: the diatom is therefore located outside the helium cluster at approximately 10 a.u. of distance from it. This result confirms the "classical" predictions of Sec. IV A, the earlier calculations of Ref. 5, and the resolved electronic spectra of Ref. 6.

If we now look at the angular distributions of the helium atoms around the center of mass of the diatom reported in Fig. 8 (we have also reported the angular distribution that we get from an exploratory calculation carried out with $15{ }^{4} \mathrm{He}$ atoms in the cluster), we can further see that the quantum aggregate is not really an isotropic structure. For all clusters considered here, in fact, the helium atoms tend to locate themselves along the bond axis of the molecule (where the Jacobi angle is larger than zero) and not at the edges of the molecules (where the Jacobi angle is zero). This kind of anisotropic arrangement of the helium atoms strengthens once again the picture of a droplet or cluster structure in which the molecule lies on its surface, placed horizontally on it in order to optimize its rather small interaction energy with the ${ }^{4} \mathrm{He}$ droplet. Moreover, while for the smaller clusters the distribution is fairly simple and monotonic, some structure

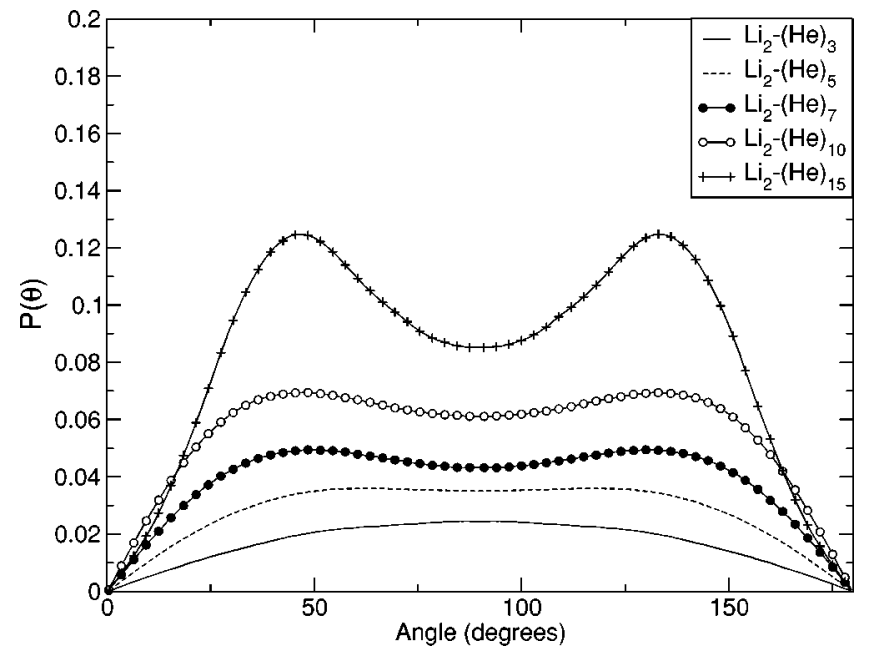

FIG. 8. Angular distributions of the helium atoms around the diatomic center of mass.

appears in the cluster when increasing the size beyond $5{ }^{4} \mathrm{He}$ atoms. In particular, a density distribution maximum appears at angles of $\sim 40^{\circ}-50^{\circ}$ for $n \geqslant 10$. It can be viewed as the effect of the incipient formation of the "dimple" that has been surmised to exist in the larger droplets. ${ }^{5}$

Although both the classical calculations examined in Sec. IV A and the quantum structures discussed in Sec. IV B provide a complex shape in which the molecules resides "outside" the helium cluster, they differ in the way the molecule is placed with respect to the helium atoms. In the classical minimization structures, in fact, the molecule points perpendicularly the "surface" of the helium atoms, while the quantum results lead us to view the molecule oriented parallel to the surface. The difference is due to the fact that the interaction PES of the $\mathrm{Li}_{2}-\mathrm{He}$ system, although rather weak, is nevertheless anisotropic and has its deepest minimum in the collinear geometry. A classical minimization will therefore favor the structures in which all the helium atoms reside on one side of the diatomic molecule. However, as we have seen in our quantum DMC calculations, the ZPE associated with the bound structures is very large and therefore the true bound states are localized on the top of the potential wells where they are largely insensitive to the small "corrugation" of the well region below (see Fig. 2). In the quantum mechanical calculations therefore the molecule lies parallel to the surface of the cluster in such a way as to optimize the small interaction energy that exists in the present systems.

\section{PRESENT CONCLUSIONS}

We have described here a newly computed interaction potential energy surface for $\mathrm{Li}_{2}-\mathrm{He}$ obtained at the QCISD(T) level, using quadruple-zeta basis sets. We have employed the rigid rotor part of the potential energy surface to generate the total interaction used to describe the forces acting within $\mathrm{Li}_{2}-\mathrm{He}_{n}$ clusters, here viewed as a sum of two-body contributions. In such systems in fact, the threebody terms have been seen as being almost negligible. We have then calculated realistic quantum mechanical nuclear energies and wave functions by using a DMC approach to 
the solution of the Schrödinger equation. Although we have analyzed only a few of the possible clusters, we have been able to extract from our results useful information on the structure and energies of such special aggregates. In particular, we have seen that the binding energy of the $\mathrm{Li}_{2}$ molecule to a ${ }^{4} \mathrm{He}$ atom is extremely small and, as expected, the presence of the alkali diatom is to be considered as a nearly negligible perturber of pure bosonic helium clusters of the same size. With respect to the experimental findings of Ref. 6 , we may conclude that the difficulties in detecting the spectra of the singlet molecule are most certainly due to the presence of such weak binding energies, a feature which makes any dynamical process taking place on the droplet, or in the droplet, capable of overcoming them, thereby making the molecule detach from the cluster's surface.

We have also tried to represent the structural properties of these weakly interacting aggregates by further taking into account the fact that a classical picture in terms of localized nuclei is certainly inaccurate when the ZPE values are more than $90 \%$ of the total binding energy. By analyzing the radial distributions of the atoms around the geometrical center of the cluster and the distributions of the helium atoms around the center of mass of the molecule, we have further confirmed the conclusion that the molecule is to be considered as placed outside the cluster and oriented parallel to its "surface." Following the suggestion of the experimental findings in Ref. 6, we are currently repeating the calculation for a molecule in its triplet state, a situation where the title molecule should present a different strength of its interaction with the helium atoms of the droplet.

\section{ACKNOWLEDGMENTS}

The financial support of the University of Rome "La Sapienza" Scientific Committee, of the CASPUR Supercomputing Center, and of the INFM Institute is gratefully acknowledged. We also thank the Agnelli Foundation for the award of an exchange grant with Koc University.
${ }^{1}$ K. B. Whaley, in Advances in Molecular Vibrations and Collision Dynamics, edited by J. Bowman (JAI, Stamford, CT, 1998), Vol. 3, pp. 397-451.

${ }^{2}$ J. P. Toennies and A. F. Vilesov, Annu. Rev. Phys. Chem. 49, 1 (1998).

${ }^{3}$ Y. Kwon, P. Huang, M. V. Patel, D. Blume, and K. B. Whaley, J. Chem. Phys. 113, 6469 (2000).

${ }^{4}$ F. Stienkenmeier, J. Higgins, C. Callegari, S. Kanorsky, W. E. Ernst, and G. Scoles, Z. Phys. D: At., Mol. Clusters 38, 253 (1996).

${ }^{5}$ F. Dalfovo, Z. Phys. D: At., Mol. Clusters 29, 61 (1994).

${ }^{6}$ F. Stienkenmeier, W. E. Ernst, J. Higgins, and G. Scoles, J. Chem. Phys. 102, 615 (1995).

${ }^{7}$ F. Stienkenmeier, J. Higgins, W. E. Ernst, and G. Scoles, Phys. Rev. Lett. 74, 3592 (1995).

${ }^{8}$ M. Fuchs and J. P. Toennies, J. Chem. Phys. 85, 7062 (1986).

${ }^{9}$ H. G. Rubahn, J. Chem. Phys. 92, 5384 (1990).

${ }^{10}$ H. G. Rubahn and K. Bergmann, Annu. Rev. Phys. Chem. 41, 735 (1990).

${ }^{11}$ F. A. Gianturco, S. Serna, G. Delgado-Barrio, and P. Villareal, J. Chem. Phys. 95, 5024 (1991).

${ }^{12}$ M. J. Frisch, G. W. Trucks, H. B. Schlegel et al., Gaussian 98, Revision A.7, Gaussian, Inc., Pittsburgh, PA, 1998.

${ }^{13}$ E. Bodo, F. A. Gianturco, F. Sebastianelli, E. Yurtsever, and M. Yurtsever, Theochem (to be published).

${ }^{14}$ D. Cvetko, A. Lansi, A. Morgante, F. Tommasini, P. Cortona, and M. G. Dondi, J. Chem. Phys. 100, 2052 (1994).

${ }^{15}$ J. Yuan and C. D. Lin, J. Phys. B 31, L637 (1998).

${ }^{16}$ I. Baccarelli, G. Delgado-Barrio, F. A. Gianturco, T. Gonzalez-Lezana, S. Miret-Artes, and P. Villarreal, Phys. Chem. Chem. Phys. 2, 4067 (2000).

${ }^{17}$ L. D. Landau and E. M. Lifshitz, Quantum Mechanics, 3rd ed. (Butterworths-Heinemann, London, 1991), Vol. 3.

${ }^{18}$ F. Sebastianelli, F. A. Gianturco, and E. Yurtsever, Int. J. Mass Spectrom. Ion Processes 220, 193 (2002).

${ }^{19}$ D. M. Ceperly and B. Alder, Science 231, 555 (1986).

${ }^{20}$ W. A. Lester and B. L. Hammond, Annu. Rev. Phys. Chem. 41, 283 (1990)

${ }^{21}$ R. N. Barnett, R. J. Reynolds, and W. A. Lester, J. Comp. Phys. 96, 258 (1991).

${ }^{22}$ M. Lewerenz and R. O. Watts, Mol. Phys. 81, 1075 (1994).

${ }^{23}$ J. B. Anderson, Int. Rev. Phys. Chem. 14, 85 (1995).

${ }^{24}$ M. Lewerenz, J. Chem. Phys. 104, 1028 (1996).

${ }^{25}$ F. Paesani, F. A. Gianturco, M. Lewerenz, and J. P. Toennies, J. Chem. Phys. 111, 6897 (1999).

${ }^{26}$ F. Paesani, F. A. Gianturco, M. Lewerenz, and J. P. Toennies, J. Chem. Phys. 112, 2239 (2000).

${ }^{27}$ F. Paesani, F. A. Gianturco, and K. B. Whaley, J. Chem. Phys. 115, 10225 (2001).

${ }^{28}$ F. Paesani and F. A. Gianturco, J. Chem. Phys. 116, 10170 (2002).

${ }^{29}$ R. A. Aziz, F. R. McCourt, and C. C. K. Wond, Mol. Phys. 61, 1487 (1987).

${ }^{30}$ M. Lewerenz, J. Chem. Phys. 106, 4596 (1997). 